\title{
Severe Sepsis in Managed Care: Analysis of Incidence, One-Year Mortality, and Associated Costs of Care
}

\author{
LEEANN BRAUN, MPH; AYLIN ALTAN RIEDEL, PhD; and LIESL MARIE COOPER, PhD
}

\begin{abstract}
OBJECTIVE: To determine severe sepsis (SS) incidence, hospital mortality, 1-year mortality, and costs associated with care in a sample of enrollees in a nationally representative individual practice association (IPA)-network managed care organization (MCO).

METHODS: This was a retrospective analysis of administrative claims data for commercial (not managed Medicare) members. We identified MCO members hospitalized for SS between July 1995 and December 1998. SS cases were identified by a combination of ICD-9-CM codes for infection and organ dysfunction. Enrollment information, physician, facility, and pharmacy claims were analyzed. Subjects with continuous enrollment were followed for 1 full year of observation. Costs were health plan payments to providers, after subtraction of member cost-share amounts.

RESULTS: The incidence rate was 0.91 cases of SS per 1,000 enrollees, increasing with age. The mean age of SS patients was 50 years, and $53 \%$ were male. Approximately $63 \%$ received surgical intervention. Mortality was $21 \%$ during the first hospitalization and $36.1 \%$ at 1 year. During follow-up, $47.1 \%$ of survivors were rehospitalized. Mean index hospitalization length of stay and costs were 16 days and $\$ 26,820$, with 1 -year inpatient and outpatient costs totaling $\$ 48,996$. Mean outpatient costs per survivor were $\$ 8,363$, and mean per-patient-permonth (PPPM) outpatient costs were $\$ 906$. Total follow-up costs including rehospitalization were similar for nonsurvivors compared with survivors $\mathbf{( \$ 7 , 7 1 0}$ versus $\$ 8,522, P=0.274)$, but PPPM costs were higher for nonsurvivors $(\$ 1,760$ versus $\$ 699, P<0.001)$.
\end{abstract}

CONCLUSIONS: Incidence, hospital, and 1-year mortality rates were lower in this population compared with literature reports and were associated with a lower average age in this managed care population. Mean SS hospitalization costs were high, and nearly one half of survivors required rehospitalization within 1 year. Study results suggest the need to evaluate SS interventions for improvement in health outcomes and cost outcomes, particularly in postsurgical patients.

KEYWORDS: Managed care, Severe sepsis, Outcomes, Resource use, Incidence, Mortality, Epidemiology

J Manag Care Pharm. 2004;10(6):521-30

Authors

LEEANN BRAUN, MPH, is associate clinical development consultant, Global Clinical Operations, and LIESL MARIE COOPER, PhD, is director, U.S. Outcomes Research, Eli Lilly and Company, Indianapolis, Indiana; AYLIN ALTAN RIEDEL, $\mathrm{PhD}$, is senior researcher, Ingenix Inc., Eden Prairie, Minnesota.

AUTHOR CORRESPONDENCE AND REQUEST FOR REPRINTS:

LeeAnn Braun, MPH, Associate Clinical Development Consultant, Global Clinical Operations, Eli Lilly and Company, Indianapolis, IN 46285. Tel: (317) 277-6157; Fax: (317) 276-8206; E-mail: braun_leeann@lilly.com

Copyright $\odot$ 2004, Academy of Managed Care Pharmacy. All rights reserved.
$\mathrm{W}$ ith an estimated 750,000 cases of severe sepsis (SS) in 1995 and concern about increasing incidence, sepsis and its related sequelae continue to be a significant health care challenge in the United States. ${ }^{1}$ Despite technological advances to better understand pathophysiology and treatment of sepsis, few studies report incidence, mortality, and economic burden. To our knowledge, only 1 recent study addresses SS (incidence) and related outcomes (mortality and cost) in a nationally representative sample; however, it does not provide results of long-term mortality and resource use by insurance status. ${ }^{1}$ While managed care enrollment exceeds 177 million members in the United States, the characteristics of SS in this population are unknown. ${ }^{2}$

Given the paucity of data on SS in the managed care environment, the objective of this study is to explore the incidence, demographics, cost, and mortality of SS in a managed care environment. We applied the algorithm suggested by Angus et al. to retrospective claims from a large representative sample from 16 individual practice association (IPA) managed care plans. ${ }^{1}$ For the purpose of data evaluation for our readers, similar results from Angus et al. were used as a "frame of reference" comparator. To our knowledge, the results reported here represent the first descriptive study of SS in this payer population. It is important to review existing sepsis terminology and epidemiological results to best interpret these findings.

\section{Sepsis Terminology}

SS is generally recognized as an acute systemic response to infection resulting in inflammation and, ultimately, organ dysfunction. The terms septicemia, sepsis, severe sepsis, sepsis syndrome, and septic shock, among others, have been used interchangeably to describe the sepsis continuum. The lack of uniformly accepted definitions has made it difficult to interpret and describe the epidemiological impact of sepsis on society. In 1992, a consensus conference sponsored by the American College of Chest Physician (ACCP) and the Society of Critical Care Medicine (SCCM) proposed a uniform set of definitions to improve bedside detection, standardization of research protocols, and improved dissemination and application of information from clinical and epidemiological studies. ${ }^{3}$ Again, in December 2001, a 30-member sepsis-working group formed by ACCP/SCCM met to review current definitions (Table 1). ${ }^{4}$ Due to the lack of additional, clear, and precise data, current definitions remain unchanged. It is important to understand these definitions and use them as intended to provide the context for diagnosis, patient care, and future research. 


\section{TABLE 1}

\section{Definitions of the ACCP/SCCM Consensus Conference ${ }^{3}$}

Infection: microbial phenomenon characterized by an inflammatory response to the presence of microorganisms or the invasion of normally sterile host tissue by those organisms

Bacteremia: the presence of viable bacteria in the blood

Systemic inflammatory response syndrome (SIRS): the systemic inflammatory response to a variety of several clinical insults. The response is manifested by 2 or more of the following conditions:

1. temperature $>38^{\circ} \mathrm{C}\left(100.4^{\circ} \mathrm{F}\right)$ or $<36^{\circ} \mathrm{C}\left(96.8^{\circ} \mathrm{F}\right)$

2. heart rate $>90$ beats per minute

3. respiratory rate $>20$ breaths per minute or $\mathrm{PaCO}_{2}<32$ torr $(4.3 \mathrm{kPa})$

4. $\mathrm{WBC}>12,000$ cells $/ \mathrm{mm}^{3},<4,000 / \mathrm{mm}^{3}$, or $>10 \%$ immature (band) forms

Sepsis: the systemic response to infection, manifested by 2 or more of the SIRS criteria listed above

Severe sepsis: sepsis associated with organ dysfunction, hypoperfusion, or hypotension. Hypoperfusion and hypotension abnormalities may include, but are not limited to, lactic acidosis, oliguria, or acute alteration in mental status.

Hypotension: a systolic blood pressure of $<90 \mathrm{~mm} \mathrm{Hg}$ or a reduction $>40 \mathrm{~mm} \mathrm{Hg}$ from baseline in the absence of other causes of hypotension

Multiple organ failure syndrome: presence of altered organ function in an acutely ill patient such that homeostasis cannot be maintained without intervention

ACCP $=$ American College of Chest Physician; $S C C M=$ Society of Critical Care Medicine.

\section{Epidemiology: Incidence, Mortality, Long-term Outcomes, and Cost}

There is a paucity of literature reporting incidence, mortality, longterm outcomes, and associated costs of care for SS. Although there have been new International Classification of Diseases, 9th Revision, Clinical Modification (ICD-9-CM) diagnostic and procedure codes added in recent years in response to increasing interest in tracking SS treatments and associated outcomes, it remains an understudied area. While using coding algorithms to pattern SS, definitions will miss patients where coding is incomplete. However, the selected (coded) patients have been shown to have a clinical course similar to patients studied prospectively ${ }^{1,5}$

Important trends may be observed in descriptive administrative research. Despite the dearth of literature, a few reports provide a broad description of SS rates and associated costs of care. Hospital incidence is estimated to be 2 to 4 cases per 100 discharges, with resulting mortality of $30 \%$ to $50 \% .{ }^{1}$ Due to the frequent manifestation of underlying comorbidities identified in SS populations, incidence and mortality may be increased relative to those SS patients without significant comorbid illness. ${ }^{6-9}$ Because reported incidence is believed to increase rapidly beyond the age of 60 years, a generally younger, healthier managed care population would be expected to experience less-severe sepsis. However, as patient case-mix changes and managed Medicare membership increases, evaluation of underlying comorbidity may be considerably more important when determining appropriate patient care.

Few studies address long-term outcomes for those who have survived a sepsis episode. It was estimated in a Veterans Affairs cohort that sepsis increased the risk of death in the first year following the acute event and probably for as long as 5 years. The $50 \%$ reduction in lifetime survival was determined based on a decrease from 8 to 4 years. ${ }^{9}$ The magnitude of survival reduction may be smaller in a younger sample and should be evaluated in a more contemporary critical care environment.

In 2003, epidemiological analyses were conducted by Martin et al. using the National Hospital Discharge Survey (nonfederal acute care hospital data). While the primary focus of this study was to determine the occurrence of all sepsis cases (e.g. septicemia and SS) in the United States from 1979-2000, SS subanalyses were conducted as well. Over the 21-year period, an $11 \%$ increase in SS was observed, and organ failure occurred more frequently (34\%), with a cumulative effect on mortality. Reported outcomes by Martin et al. support and validate existing published literature. $^{5}$

A managed care population with continuous eligibility allows for estimation of 1-year (3, 6, 12 months) survival as well as subsequent hospitalization and health care costs. We chose to make comparisons of hospital costs and resource use of SS patients with those from Angus et al., given the similar end points evaluated. These 1995 data reported by Angus et al. showed average hospital costs of $\$ 22,100$, increasing to $\$ 29,900$ for patients requiring an intensive care unit (ICU) stay and $\$ 30,800$ for surgical patients. ${ }^{1}$ Mortality and costs associated with SS affect patients, hospitals, and payers. With a focus on improved patient care and mounting budget pressures, hospitals and managed care organizations (MCOs) will be increasing the attention given to the care of patients with SS.

The incidence, mortality, and cost of SS are high in the United States. With managed care demographics changing and enrollment increasing, further research evaluating the characteristics of SS in diverse patient groups is needed.

\section{Methods}

This study was a retrospective, administrative claims analysis using enrollment information, physician, facility, and pharmacy claims from enrollees in 16 IPA network plans (does not include managed Medicare) located in various states throughout the Midwest, Northeast, Southeast, and Western United States. This study used data for services or products provided to these MCO enrollees between July 1, 1995, and December 31, 1999. Total health plan enrollment during the time period used to identify study patients (July 1, 1995, to December 31, 1998) was 5.4 million. Data from the National Death Index (NDI) for the years 1995-1999 were used to confirm inpatient hospitalization and to identify death outside of hospitalization. In the case of death outside the hospital stay, the NDI data provide both identification and confirmation of patient death. 


\section{Population}

Enrollees were included as study patients if they were hospitalized for SS at least once during the patient identification period. SS was defined based on the presence of ICD-9-CM diagnosis codes for both infection and organ dysfunction during the same hospitalization. We used a modified list of ICD-9-CM codes from Angus et al. to eliminate relatively infrequent and unusual infection codes. ${ }^{1}$

Patients were retained for analysis if their health plan coverage included a pharmacy benefit and if they were continuously enrolled for the 6 months prior to their index hospitalization and until their death or the end of a 365-day period following the index admission date (whichever occurred earlier). NDI data were used to confirm in-hospital deaths and identify patient death during the remaining postdischarge period.

Administrative claims information regarding health services costs and utilization for the 6 months prior to admission for the index hospitalization for SS and for up to 365 days following the admission date of the index hospitalization for SS were used in the analysis.

The incidence of hospitalization for SS during the patient identification period was calculated (per 1,000 health plan enrollees) prior to the application of continuous enrollment or pharmacy benefit requirements.

\section{Measures}

Several measures characterizing the index hospitalization for SS were defined. Site(s) of organ dysfunction (cardiovascular, respiratory, hepatic, renal, neurologic, hematologic) were defined based on ICD-9-CM codes in the primary or secondary position on hospital claims. Surgical and medical status was determined based on the presence of ICD-9-CM or current procedural terminology (CPT) codes. ${ }^{1}$ Length of stay (LOS) was calculated and the discharge diagnostic-related group (DRG) identified. Time in either an ICU or coronary care unit (CCU) was calculated, and status at discharge from the index hospitalization for SS was identified using hospital claims. Comorbid conditions present during the index hospitalization were identified based on the presence of ICD-9-CM diagnosis codes on hospital claims (Table 2). ${ }^{10,11}$

Multiple measures of health service costs and utilization were identified to describe hospitalization costs for this SS sample. Defined costs are "costs-paid" data, which represent costs paid to the provider by the health plan, after subtraction of copays and deductibles paid by the patient; none of these patients were enrolled in provider-capitated IPA health plans. Total costs for the index hospitalization for SS were also calculated for all patients. For patients who survived the index hospitalization for SS, the following cost and utilization variables were calculated for the period following discharge: number of rehospitalizations for any reason, number of rehospitalizations with infection and organ failure (suggesting

\section{TABLE 2 ICD-9-CM Codes Used to Identify Comorbidities During the Hospital Stay}

\begin{tabular}{l|c}
\hline Comorbidity & $\begin{array}{c}\text { ICD-9-CM } \\
\text { Codes Included }\end{array}$ \\
\hline Infectious and parasitic diseases & $001-139$ \\
Neoplasms & $140-239$ \\
Endocrine, nutritional, and metabolic diseases & $240-279$ \\
Diseases of blood and blood-forming organs & $280-289$ \\
Mental disorders & $290-319$ \\
Diseases of the nervous system and sense organs & $280-289$ \\
Disease of the circulatory system & $390-459$ \\
Disease of the respiratory system & $460-519$ \\
Diseases of the genitourinary system & $580-629$ \\
Complications of pregnancy, childbirth, puerperium & $630-677$ \\
Diseases of the skin and subcutaneous tissue & $680-709$ \\
Diseases of the musculosketetal system and & $710-739$ \\
connective tissue & \\
Congenital anomalies & $740-759$ \\
Certain conditions originating in the perinatal period & $760-779$ \\
Injury and poisoning & $830-999$ \\
\hline ICD-9-CM=International Classification of Diseases, 9th Revision, Clinical \\
Modification.
\end{tabular}

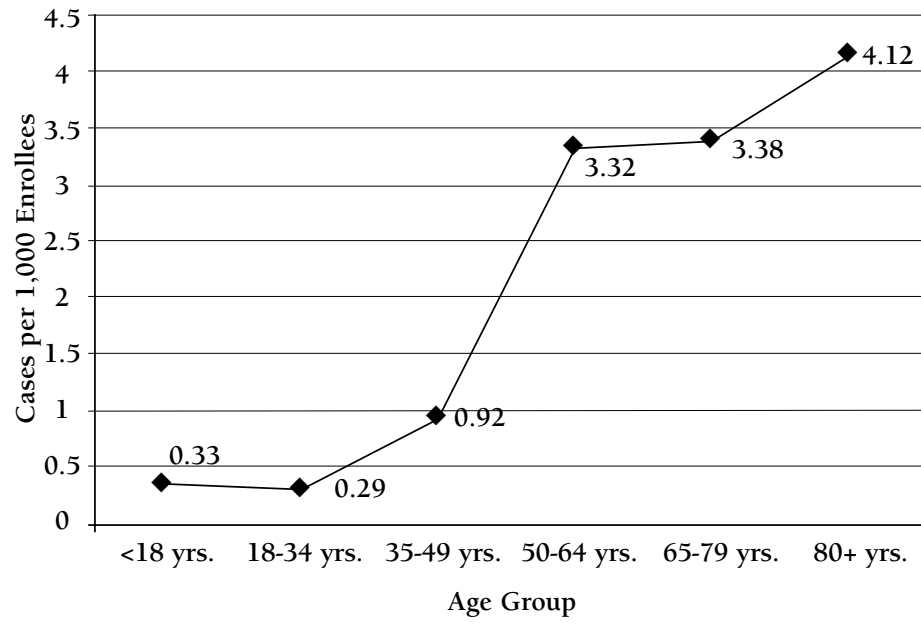

Note: Age-specific incidence rates per 1,000 were highest in older age groups, increasing from less than 1 per 1,000 enrollees in patients under age 50 years to 3 to 4 per 1,000 in patients aged years 50 and older. Incidence was calculated prior to application of continuous enrollment requirements $(n=4,856)$. 
(chi square $\left[\mathrm{X}^{2}\right]=118.79, P<0.001$ ). Table 5 details the odds of death for each age band. ORs in the table are the odds of death in the age band compared with the odds of death among patients in the 0 to 17 years age band. Not presented in this table are results that indicate that, with the exception of the 18 to 34 versus 0 to 17 year-olds, each successive age band has a significantly higher odds of death $(P<0.0001$; not shown $)$ than the previous band.

Hospital resource use and costs. Mean hospital LOS and costs for patients during the index hospitalization were 16.1 days, with an average cost of $\$ 26,820( \pm 55,900)$ higher costs for those with surgical intervention ( $\$ 38,036$ versus $\$ 7,532, P<0.001)$ and hospital nonsurvivors $(\$ 36,602$ versus $\$ 24,287, P<0.001$ ) (Tables 6 and 7 ). During the index hospitalization, there was no statistically significant difference between males and females in LOS or costs (16.3 days versus 15.6 days, $P=0.425$ and $\$ 25,900$ versus $\$ 27,631, P=0.41$ ). LOS for the first hospitalization differed by age, with patients in the youngest age band ( $<18$ years) having significantly longer LOS than the oldest age band (80+ years, 18.2 days versus 11.5 days, $P=0.012$ ). However, no differences in LOS were observed across other age bands. Patients in the youngest age band ( $<18$ years) had costs for the index SS hospitalization $(\$ 56,629)$ that were significantly higher than costs for all other age bands. Hospitalization costs for patients in the 18 to 34 , 35 to 49 , and 50 to 64 years age bands $(\$ 34,576, \$ 29,371$, and $\$ 25,678$, respectively) were significantly higher than costs for patients in the 65 to 79 and $80+$ years age bands $(\$ 11,697$ and $\$ 4,574$, respectively) (REGW, $P<0.05$ ), although this may be due, in part, to coordination of benefits with Medicare for patients aged 65 years and older. As defined in the "Measures" section, the costs included in this study are costs paid to the provider by the health plan after subtraction of member copays and deductibles. Medicare-covered costs would, therefore, be excluded.

Mean hospital LOS and costs for patients who spent time in an ICU and/or CCU during the index hospitalization were 20 days and $\$ 36,218$, and the difference in costs among nonsurvivors $(\$ 41,074)$ and survivors $(\$ 34,420)$ was not statistically significant $(P=0.059)$. Surgical patients had significantly longer LOS (Figure 2) and higher costs (Figure 3) than medical patients during the index hospitalization (21.1 days versus 7.2 days, $P<0.0001$ and $\$ 38,036$ versus $\$ 7,532, P<0.0001)$ across all age bands, with higher costs for nonsurvivors ( $\$ 45,096$ versus $\$ 35,766, P=0.011$ ).

Status upon discharge. The patient status field on hospital claims indicates status upon discharge, and patient death was verified by the NDI. Approximately $21 \%$ of patients died during the index SS hospitalization. The discharge status of $2 \%$ of patients was unknown. Fifty-one percent were discharged to home or self care, 13\% were discharged to home with home care, and 13\% were discharged or transferred to another facility (Figure 4).

\section{TABLE 5 Odds of Death During the 12-Month Study Period by Age}

\begin{tabular}{l|c|c|c|c}
\hline $\begin{array}{l}\text { Age Group } \\
\text { (Years) }\end{array}$ & $\begin{array}{c}\mathbf{N} \\
(2,834)\end{array}$ & $\begin{array}{c}\text { Number } \\
\text { of Deaths (\%) }\end{array}$ & $\begin{array}{c}\text { Odds } \\
\text { of Death }\end{array}$ & 95\% CI \\
\hline $0-17$ & 196 & $27(13.8)$ & 1.00 & - \\
$18-34$ & 263 & $48(18.3)$ & 1.40 & $(0.83-2.34)$ \\
$35-49$ & 743 & $255(34.3)$ & 3.27 & $(2.10-5.08)$ \\
$50-64$ & 1,165 & $466(40.0)$ & 4.17 & $(2.71-6.42)$ \\
$65-79$ & 383 & $176(46.0)$ & 5.32 & $(3.30-8.59)$ \\
$80+$ & 84 & $51(60.7)$ & 9.67 & $(4.89-19.13)$ \\
\hline
\end{tabular}

CI= confidence interval.

Notes: (1) All age groups $>17$ years were compared with patients in the 0-17 age group. (2) Odds of death during 12-month study period significantly increases with age $\left(X^{2}=118.79 ; P<0.001\right)$.

\section{TABLE $6 \longdiv { \text { Per-Patient Health Plan Costs Before and } }$ During Severe Sepsis Hospitalization}

\begin{tabular}{l|c|c}
\hline Cost Category $(\mathrm{N}=\mathbf{2 , 8 3 4 )}$ & Mean $(\mathrm{SD})(\$)$ & Median $(\$)$ \\
\hline $\begin{array}{l}\text { Medical costs (6 months prior to } \\
\text { severe sepsis hospitalization) }\end{array}$ & $47,622( \pm 79,790)$ & 17,418 \\
\hline Costs of first severe sepsis & $26,820( \pm 55,900)$ & 8,024 \\
hospitalization & $36,218( \pm 66,832)$ & 12,693 \\
$\quad$ Patients with ICU stay $(\mathrm{n}=1,817)$ & $10,030( \pm 17,014)$ & 5,111 \\
Patients with no ICU stay ( $=1,017)$ & $38,036( \pm 67,011)$ & 14,930 \\
Surgical patients $(\mathrm{n}=1,792)$ & $7,532( \pm 13,751)$ & 4,516 \\
\hline Nonsurgical patients $(\mathrm{n}=1,042)$ &
\end{tabular}

Notes: (1) Hospitalization costs are unadjusted for inflation and were collected for the period of July1, 1995, to December 31, 1998. (2) The variable presentation of severe sepsis in each patient contributes to the variable per-patient cost. Mean per-patient cost outcomes represent such variability.

ICU $=$ intensive care unit.

\section{Postindex Hospitalization Follow-up Period}

Twelve-month resource use and costs. While many studies may use medians to describe "typical patient costs," we chose to use means as the primary description and median values as the secondary description of the data. This was done to facilitate better understanding of the variability of cost and LOS due to high acuity and mortality. Mean costs appear highly skewed compared with the median costs. However, given the highly variable SS disease process and high mortality, we believe mean costs best represent the "average" for this patient population.

Patients were followed for 12 months from hospital admission or until death, whichever occurred first. Various costs and utilization variables were analyzed by survival status during the postdischarge period. The mean number of all-cause rehospitalizations and associated costs were higher for nonsurvivors than survivors (1.33 hospitalizations versus 0.94 hospitalizations, $P<0.001$ and $\$ 25,976$ versus $\$ 11,854$, $P<0.001$.) Mean outpatient visit costs were not significantly different between survivors and nonsurvivors ( $\$ 5,862$ versus 


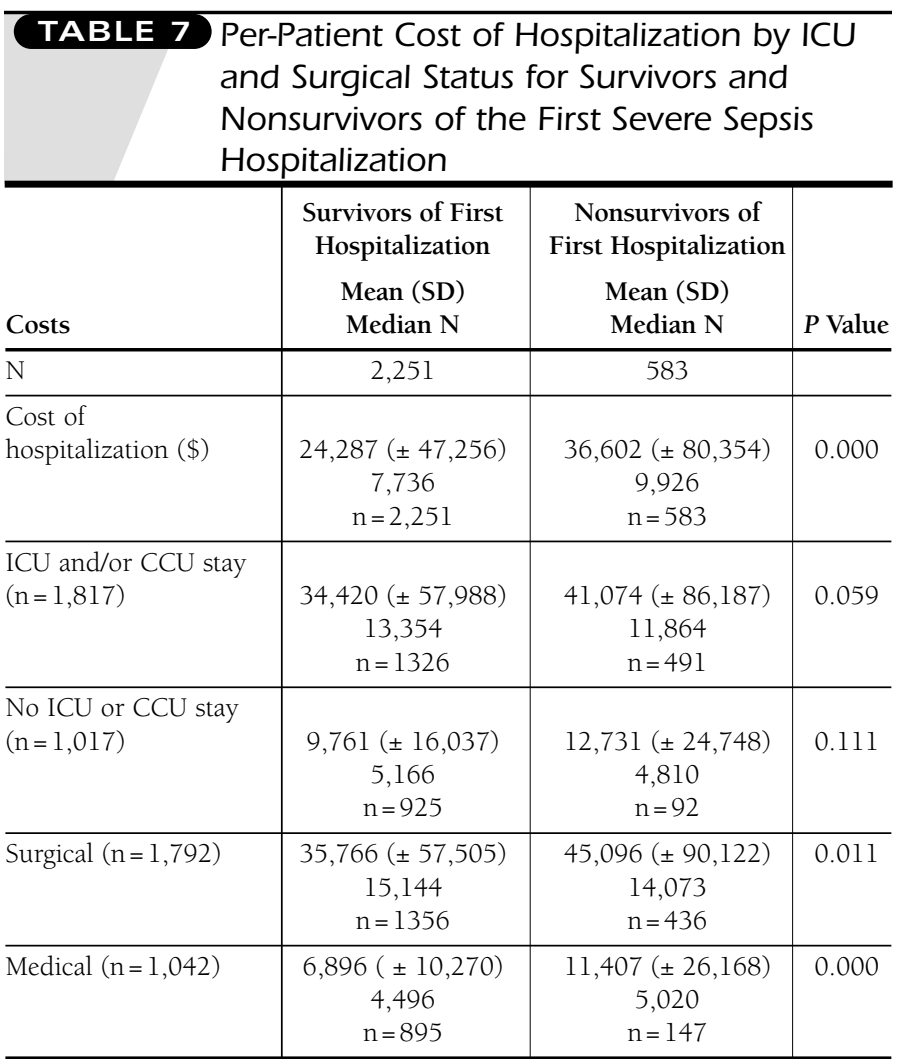

$I C U=$ intensive care unit; $C C U=$ cardiac care unit.

Notes: (1) The variable presentation of severe sepsis in each patient contributes to the variable per-patient cost. Mean per-patient cost outcomes represent such variability. (2) Hospitalization costs are unadjusted for inflation and were collected for the period from July 1, 1995, to December 31, 1998.

$\$ 5,882, P=0.976)$ although the number of outpatient visits was significantly greater for survivors because of increased opportunity to attend visits ( 30.7 visits versus 20.4 visits, $P<0.001$ ). Mean total outpatient costs (sum of costs for ER visits, costs for outpatient visits, and pharmacy costs) were similar for survivors and nonsurvivors $(\$ 8,522$ versus $\$ 7,710, P=0.274)$, while PPPM outpatient costs were significantly greater for nonsurvivors ( $\$ 1,760$ per month versus $\$ 699$ per month, $P<0.001)$. Postdischarge utilization by survival status is presented in Table 8

Subjects who died during the study period had less opportunity (time) to seek outpatient care and therefore had lower total outpatient costs. Medical costs, (the costs of all nonpharmacy health services: ER costs + outpatient visit costs + inpatient visit costs) during the postdischarge period were significantly higher for nonsurvivors than survivors $(\$ 32,474$ versus $\$ 18,425, P<0.001$ ). PPPM outpatient costs, outpatient visit costs, and pharmacy costs were lower for survivors than for nonsurvivors. One-year inpatient (including index hospitalization) and outpatient costs totaled $\$ 48,996$.

\section{Discussion}

SS affects a diverse, heterogeneous population. Such heterogeneity can lead to variable diagnosis, treatment, cost, and clinical outcomes. Until recently, attention regarding SS has been given to older populations, particularly over the age of 65 years receiving Medicare benefits. Although incidence increases with advancing age, younger patients do develop SS. Given a generally younger and healthier demographic profile in non-Medicare-managed care plans, descriptive study of SS in such populations may elucidate risk patterns that assist in planning for clinical care of affected patients. In 1999, the estimated average cost of all-cause hospital admissions from this MCO's commercial population was $\$ 6,160$, while the average SS hospital admission cost was $\$ 28,820$. Clearly, SS consumes significant resources even in a younger, managed care population. With changing case-mix due to shifting demographics as the population ages, MCOs may see increased coordination of benefits with Medicare, meaning patients will draw benefits from 2 sources: a managed care supplement and from Medicare. Combined with availability of new technologies, these factors make understanding of changes in disease incidence, resource use, and costs of care of increasing interest in the administrative and clinical environment.

SS incidence (0.91 per 1,000), in-hospital mortality (21\%), and 1-year mortality (36\%) in this managed care sample were lower than rates reported in other retrospectively and prospectively defined populations. ${ }^{1,6,8,12,13}$ However, the higher rate of SS in patients who received surgical intervention, a high rehospitalization rate $(47.1 \%)$, and high hospital and follow-up costs suggest the need to further investigate the special needs of SS patients in managed care plans.

Information regarding hospital resource use and costs associated with the care of SS patients is limited. Angus et al. provide a comprehensive retrospective evaluation of resource use and costs associated with the care of SS patients in a large nationally representative sample. Consistent with our findings, Angus et al. found estimated mean length of stay for nonsurvivors and survivors to be similar, with considerably higher costs for nonsurvivors. Although results are comparable in the 2 studies, consideration must be given to the average age difference between the 2 populations (in the Angus et al. study, the average reported age was 14 years older than in our managed care sample) as well as cost reporting definitions and study time frame. Angus et al. reported costs based on total charges converted to costs using published composite cost-tocharge ratios in year 1995 only, while the costs in our sample are total plan costs, which represent the cost paid to the provider by the health plan after subtraction of member copays and deductibles, for the time period from 1995-1998.

The most recent clinical trial for SS, Recombinant Human Activated Protein C Worldwide Evaluation in Severe Sepsis (PROWESS), reported findings different from our retrospective 
study, with longer LOS and higher hospital costs for survivors. ${ }^{14}$ The differences may be attributed to several factors. All PROWESS patients received ICU care and $75 \%$ had $2+$ organ dysfunctions, while $64 \%$ of managed care patients in our study received ICU care and 30\% had 2+ organ dysfunctions. Given the high rate of organ dysfunction in the PROWESS trial, mortality may have occurred early, reducing costs compared with the generally younger managed care patients with fewer organ dysfunctions and longer LOS.

While overall LOS was similar for the 2 populations PROWESS nonsurvivors had a shorter LOS than nonsurvivors in our cohort. When PROWESS patients were compared with managed care patients in our study who received ICU care, LOS was similar for survivors, with a longer LOS for survivors in each population. However, costs remained higher for PROWESS survivors, while managed care costs were not different by survival status. Rehospitalizations were also more frequent and more costly for nonsurvivors than for survivors in the managed care cohort in our study. Again, the severity of illness as determined by number of organ failures may indicate early death and lower costs for PROWESS nonsurvivors.

When evaluating results from retrospective and prospective studies, the nature of the sample selection, cost definition, and study time frame must be kept in mind. In the PROWESS clinical trial sample, costs are total 28-day costs from randomization using itemized charges converted to costs using department cost-to-charge ratios in year 2000 dollars, which are different from the calculations in our study and in Angus et al. Further research and validation is necessary to bridge the gap in identification of SS patients and determination of the cost-of-care retrospectively versus prospectively.

ICU care is a common component of care for SS patients. While other studies report that slightly more than $50 \%$ of SS cases required ICU care, more than $60 \%$ of our managed care patients received ICU care. ${ }^{1,15}$ High SS rates in patients with surgical intervention may indicate an increased need for ICU care. The demand for more resources may be due to surgery recovery treatment norms or the direct result of the level of care required to support the SS sequelae, thereby incurring higher costs. Because temporal relationships (using ICD-9-CM coding within a hospitalization) cannot be defined in our administrative data set, explanation for the increased need for ICU care is speculative. Despite these differences, the proportion of patients with an ICU stay for this sample is similar to that seen in Angus et al. Patients with an ICU stay in each study had a total average LOS exceeding 20 days. Costs for these patients were also similar, despite the earlier date of the Angus et al. work. ${ }^{1}$ This managed care sample, however, has shorter reported average LOS and costs for those not requiring ICU care, possibly due to less variance in this younger group of patients. For patients who required either ICU or CCU care, the hospital costs were not different between

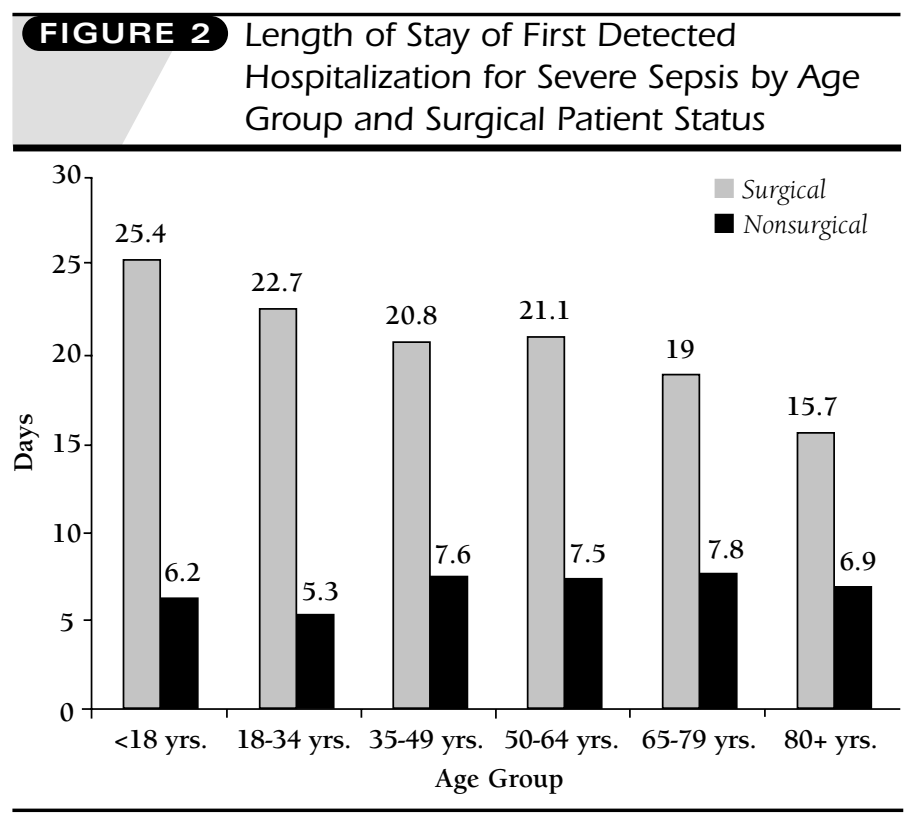

Note: Average length of stay for those in the older age bands (65+) are noticeably shorter than those of the younger age bands. Given the higher mortality in the older age bands, fewer days spent in the hospital are expected. The older age bands may also be less descriptive of the overall $65+$ population given the coordinated benefits with Medicare.

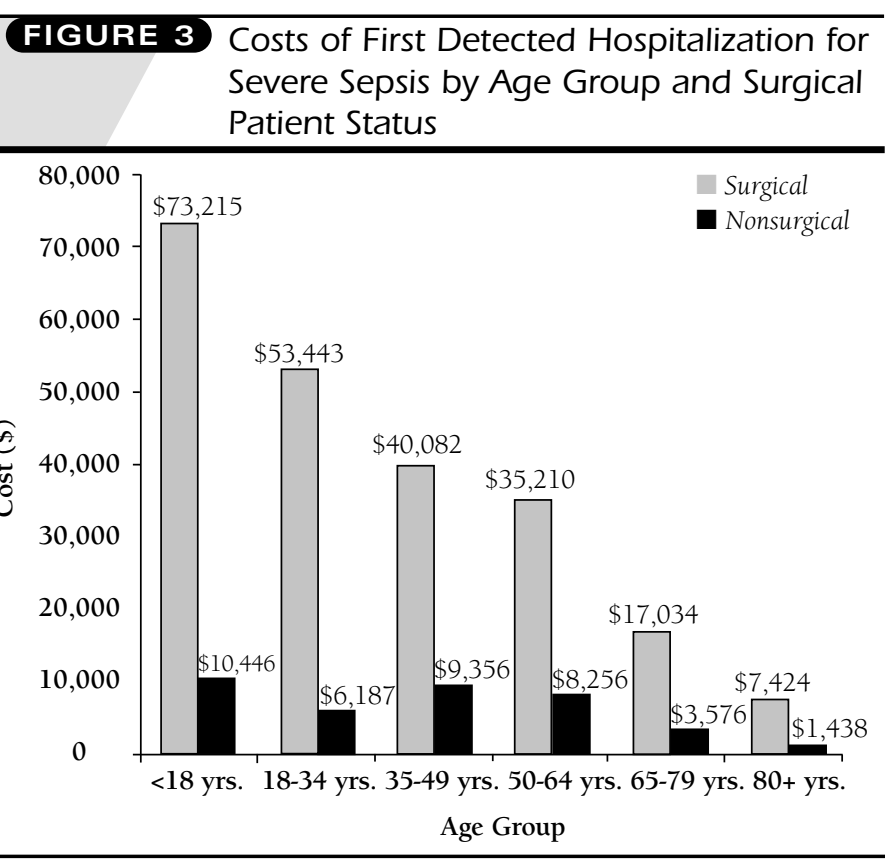

Note: The cost of the first severe sepsis hospitalization is markedly lower in the age bands $65+$ years. As mortality increases with age (Figure 1), patients spend fewer days in the hospital (Figure 2) and subsequently incur fewer costs (Figure 3). 


\section{FIGURE 4 Patient Status at Discharge from First Detected Hospitalization for Severe Sepsis}

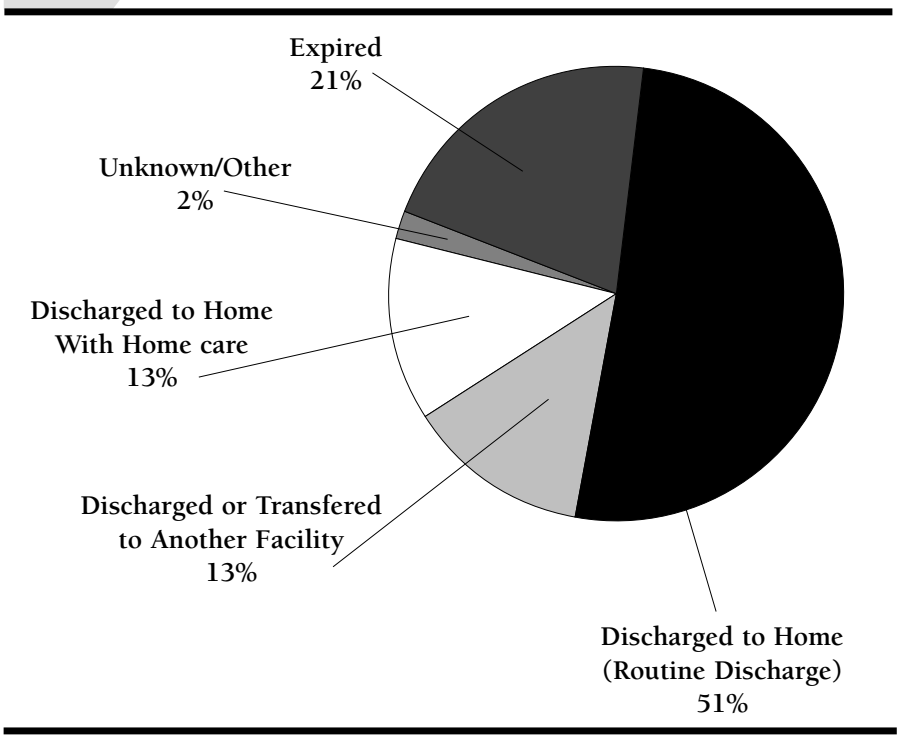

survivors (mean $\$ 34,420$ ) and nonsurvivors (mean $\$ 41,074$, $P=0.059$ ) (Table 7)

All survivors of an index hospitalization for SS have the opportunity to obtain outpatient care, incurring costs subsequent to hospitalization. However, depending on patient demographics, comorbid conditions, and severity of the septic episode, outcomes and costs may differ, particularly if the patient is near death. Such variability is represented in the difference between mean and median per-patient costs (as observed in Tables 6, 7, and 8). Because patients who died during the study had less time to seek outpatient care, it would seem that they would have lower total costs. However, their outpatient visits were statistically fewer, they had more ER visits and greater ER costs, and total outpatient costs did not differ (Table 8). Prior to the death of a nonsurvivor, however, resource demand was high, as represented in higher PPPM costs. For example, all-cause rehospitalizations and associated medical costs were higher for nonsurvivors than survivors in this managed care cohort, suggesting that the nonsurvivors were more severely ill and more resource-intensive prior to death. Due to the lack of published literature reporting long-term outcomes and costs, it is difficult to make further comparisons.

\section{Limitations}

The findings of our study are subject to the vagaries of using claims data to define a clinical condition that is hard to define prospectively and particularly difficult to define retrospectively. While temporal relationships may be identified using ICD-9-CM codes and CPT codes in hospital administrative data with automated records, we did not have access to hospital medical records or other clinical datasets to better determine the clinical detail that would allow for this determination of temporality. However, we believe the application of this algorithm is a useful proxy in the absence of a single identified code for SS, given that no single code was available when data were collected. We utilized identified ICD-9-CM codes in a managed care administrative dataset. To select patients in a more conservative method, we required that both infection and acute organ failure must occur within the same hospitalization and be coded as such. Temporality could not be identified; therefore, we do not know if the infection came before or after the organ dysfunction. We recognize that this algorithm may lead to "false positives," or inclusion of patients with coded infection and organ failure who do not have true SS; however, it is also likely that there are many "false negatives" when additional coding is limited due to space constraints or realization of reimbursement.

Ollendorf et al. challenge the use of administrative data for "sepsis" surveillance or research planning. Without a code for SS, coding may be inconsistent, as revealed in their study. ${ }^{16}$ Retrospective identification of SS without a specific code is not perfect, but it allows a more timely description and evaluation of the estimated impact of SS on a managed care sample during the initial planning and adoption period for new therapeutic advances. The relevant question to consider is how well the presence of infection and organ failure in a single hospital stay describes the resource needs in the event these identified cases do not provide adequate clinical proxy for SS. With the recent approval of an ICD-9-CM code for SS (995.92) various coding schemes can be eliminated, and accurate identification of these patients in future research is anticipated. ${ }^{17}$

Data used in our analyses were collected from 1995-1998. With the entrance of new drugs to market, such as linezolid for bacterial infections in 2000 and drotrecogin alfa activated for SS in 2001, more treatment options are now available to treat patients with infection and subsequent acute organ failure. Unfortunately, because the number of SS patients in a managed care population is relatively small, it may take several years to assess the impact of new technologies on outcomes and the use of these data in evolution of the standard of care.

Limitations are probable in the evaluation and comparison of prospective clinical research and retrospective database analyses. Study design, patient population, cost definitions, and study duration must be considered in critical evaluation. Important differences exist between this study, the Angus et al. study, and the PROWESS clinical trial. Our retrospective database study included MCO members hospitalized for SS between July 1995 and December 1998. Measured costs are total plan costs, which represent costs paid to the provider by the health plan after subtraction of member copays and deductibles (Medicare-covered costs not included). Angus et al. retrospectively studied all discharged hospital patients identified from the discharge data from 7 state hospitals in 7 U.S. states in the year 1995. Reported costs were based on total charges, 
converted to costs using published composite cost-to-charge ratios. ${ }^{1}$ In the PROWESS study, patients with SS were randomized into a double-blind, placebo-controlled clinical trial. Reported costs were total 28-day costs from randomization using itemized charges converted to costs using departmental cost-tocharge ratios in year 2000 dollars. ${ }^{13}$

Administrative claims have expected limitations. The degree to which claims data can accurately capture an individual medical history is imperfect. This data set was not designed primarily for research. While these data are excellent for understanding "real-world" patterns of utilization and cost, they are subject to possible coding errors, undercoding, and coding for the purpose of ruling out conditions (e.g., pancreatitis, acute renal failure) rather than actual disease. ${ }^{18}$ Despite its limitations, administrative data remain a useful data source to estimate answers to questions such as those arising after the introduction of a new agent. These data allow for the examination of health care utilization and expenditure patterns in a real-world setting, away from the highly controlled environment of the clinical trial.

The data used for this study come from a managed care population. Therefore, results of this analysis are primarily applicable to the treatment of severe sepsis in a managed care environment. MCO health plans included in this analysis, however, are discounted fee-for-service and not capitated or gate-keeper models. The wide geographic distribution across the United States may allow generalization to managed care populations on a national level.

\section{Conclusions}

While SS occurs less frequently in this younger, managed care population than in other reported studies, it still occurs quite frequently, and given the high cost per patient, managed care is significantly challenged by patients who develop SS. With the aging of America and the renewed interest in Medicare managed care programs, enrollment and case-mix in managed care will change. It is important to further define resource needs to improve clinical and economic outcomes. Periodic evaluation of the incidence, mortality, and costs associated with SS in a managed care environment is helpful to appropriately care for patients and to manage resources.

\section{ACKNOWLEDGMENTS}

We would like to acknowledge and thank Robert Obenchain, $\mathrm{PhD}$, senior research scientist, Eli Lilly and Company, for his expert statistical insight.

\section{DISCLOSURES}

Funding for this research was provided by Eli Lilly and Company and was obtained by authors LeeAnn Braun and Liesl Marie Cooper. Braun and Cooper are employed by Eli Lilly and Company; author Aylin Altan Riedel is employed by Ingenix Pharmaceutical Services and worked on this research under contract with Lilly. Braun served as principal author of the study. Study concept and design, analysis and interpretation of data, and statistical expertise were contributed by all authors. Drafting of the manuscript was primarily the work of Braun and Riedel, and its critical revision was the work of all authors.

\section{TABLE 8 Per-Patient Postdischarge Resource Utilization by Survival Status (Section I: Visits, Section II: Costs)}

\begin{tabular}{|c|c|c|c|}
\hline & \begin{tabular}{|c|} 
Survivors of \\
Postdischarge Period \\
Mean $( \pm$ SD $)$ \\
Median
\end{tabular} & $\begin{array}{c}\text { Nonsurvivors of } \\
\text { Postdischarge Period } \\
\text { Mean }( \pm \text { SD }) \\
\text { Median }\end{array}$ & $P$ Value \\
\hline \multicolumn{4}{|l|}{ Section I: Visits } \\
\hline$\overline{\mathrm{N}}$ & $1,811(80.4 \%)$ & $440(19.6 \%)$ & \\
\hline $\begin{array}{l}\text { Mean number of } \\
\text { rehospitalizations } \\
\text { (all-cause) }\end{array}$ & $\begin{array}{c}0.94 \pm 1.7 \\
0.0\end{array}$ & $\begin{array}{c}1.33 \pm 1.5 \\
1.0\end{array}$ & 0.000 \\
\hline $\begin{array}{l}\text { Outpatient visits } \\
\text { (number) }\end{array}$ & $\begin{array}{c}30.7 \pm 29.5 \\
22\end{array}$ & $\begin{array}{c}20.4 \pm 29.9 \\
11\end{array}$ & 0.000 \\
\hline $\begin{array}{l}\text { PPPM outpatient } \\
\text { visits (number) }\end{array}$ & $\begin{array}{c}2.51 \pm 2.42 \\
1.80\end{array}$ & $\begin{array}{c}4.34 \pm 4.42 \\
3.28\end{array}$ & 0.000 \\
\hline ER visits (number) & $\begin{array}{c}2.04 \pm 3.83 \\
1\end{array}$ & $\begin{array}{c}2.53 \pm 3.00 \\
2\end{array}$ & 0.011 \\
\hline \multicolumn{4}{|l|}{ Section II: Costs } \\
\hline Medical costs $(\$)^{*}$ & $\begin{array}{c}18,425 \pm 41,814 \\
4,765\end{array}$ & $\begin{array}{c}32,474 \pm 60,191 \\
9,503\end{array}$ & 0.000 \\
\hline $\begin{array}{l}\text { Total outpatient } \\
\operatorname{costs}(\$) \dagger\end{array}$ & $\begin{array}{c}8,522 \pm 13,421 \\
4,459\end{array}$ & $\begin{array}{c}7,710 \pm 15,949 \\
2,771\end{array}$ & 0.274 \\
\hline $\begin{array}{l}\text { Cost of outpatient } \\
\text { visits (\$) }\end{array}$ & $\begin{array}{c}5,862 \pm 12,336 \\
2,025\end{array}$ & $\begin{array}{c}5,882 \pm 15,003 \\
1,141\end{array}$ & 0.976 \\
\hline ER visit costs (\$) & $\begin{array}{c}733 \pm 1,512 \\
233\end{array}$ & $\begin{array}{c}921 \pm 2,182 \\
354\end{array}$ & 0.034 \\
\hline Pharmacy costs (\$) & $\begin{array}{c}1,927 \pm 2,967 \\
1,066\end{array}$ & $\begin{array}{c}907 \pm 1,567 \\
329\end{array}$ & 0.000 \\
\hline $\begin{array}{l}\text { PPPM outpatient costs } \\
\text { (\$ per month) }\end{array}$ & $\begin{array}{c}699 \pm 1,100 \\
366\end{array}$ & $\begin{array}{c}1,760 \pm 3,453 \\
925\end{array}$ & 0.000 \\
\hline $\begin{array}{l}\text { PPPM outpatient visit } \\
\text { costs ( } \$ \text { per month) }\end{array}$ & $\begin{array}{c}480 \pm 1,011 \\
166\end{array}$ & $\begin{array}{c}1,204 \pm 3,303 \\
377\end{array}$ & 0.000 \\
\hline $\begin{array}{l}\text { PPPM pharmacy costs } \\
\text { (\$ per month) }\end{array}$ & $\begin{array}{c}158 \pm 243 \\
87\end{array}$ & $\begin{array}{c}216 \pm 396 \\
114\end{array}$ & 0.000 \\
\hline
\end{tabular}

* "Medical costs" include cost of all nonpharmacy health services (emergency room [ER] costs + outpatient visit costs + inpatient visit costs following discharge from first hospitalization from severe sepsis).

† "Total Outpatient costs" include costs for all outpatient utilization (ER costs + outpatient visit costs + pharmacy costs).

Notes: (1) Per-patient-per-month (PPPM) costs were not calculated for "Inpatient costs" or aggregate cost category "Medical costs," which includes inpatient costs. Given that inpatient costs occur over a short period of time and tend to be high, converting them to PPPM values creates unrealistically high per-30-day cost amounts. Emergency room costs were also not converted to PPPM values for this reason. (2) The variable presentation of severe sepsis in each patient contributes to the variable per-patient cost. Mean per-patient cost outcomes represent such variability.

\section{REFERENCES}

1. Angus DC, Linde-Zwirble WT, Lidicker J, et al. Epidemiology of severe sepsis in the United States: analysis of incidence, outcome, and associated costs of care. J Crit Care Med. 2001;29:1-32.

2. Managed care fact sheets: managed care national statistics. Available at: http://www.mcareol.com/factshts/factnati.htm. Accessed March 1, 2002 
3. Anonymous. American College of Chest Physicians/Society of Critical Care Medicine Consensus Conference: Definitions for sepsis and organ failure and guidelines for the use of innovative therapies in sepsis. J Crit Care Med. 1992;20:864-74

4. Levy M. Review of Sepsis Definitions Conference. Presented at: The 31st Critical Care Congress of the Society of Critical Care Medicine; January 28, 2002; San Diego, CA.

5. Martin GS, Mannino DM, Eaton S, Moss M. The epidemiology of sepsis in the U.S. from 1979 through 2000. New Engl J Med. 2003;348:1546-54.

6. Brun-Buisson C, Doyon F, Carlet J, et al. Incidence, risk factors, and outcome of severe sepsis and septic shock in adults. A multicenter prospective study in intensive care units. French ICU Group for Severe Sepsis. JAMA. 1995;274:968-74.

7. Perl TM, Dvorak L, Hwang T, Wenzel RP. Long-term survival and function after suspected gram-negative sepsis. JAMA. 1995;274:338-45.

8. Pittet D, Thievent B, Wenzel RP, et al. Importance of pre-existing co-morbidities for prognosis of septicemia in critically ill patients. Intensive Care Med. 1993;19:265-72.

9. Quartin AA, Schein RM, Kett DH, Peduzzi PN. Magnitude and duration of the effect of sepsis on survival. Department of Veterans Affairs Systemic Sepsis Cooperative Studies Group. JAMA. 1997;277:1058-63.

10. Lee DW, Meyer JW, Clouse J. Implications of controlling for comorbid conditions in cost-of-illness estimates: a case study of osteoarthritis from a managed care system perspective. Value Health. 2001;4:329-34.
11. Hall JA, Nelson MA, Meyer JW, Williamson T, Wagner S. Costs and resources associated with the treatment of overactive bladder using retrospective medical care claims data. Manag Care Interface. 2001;14:69-75.

12. Rangel-Frausto MS, Pittet D, Costigan M, Hwang T, Davis CS, Wenzel RP The natural history of the systemic inflammatory response syndrome (SIRS). A prospective study [see comments]. JAMA. 1995;273:117-23.

13. Bernard GR, Vincent J, Laterre P, et al. Efficacy and Safety of Recombinant Human Activated Protein C for Severe Sepsis (PROWESS Study). New Engl J Med. 2001; 344(10):699-709.

14. Linde-Zwirble WT, Clermont G, Van Hout B, et al. Increased survivorship with drotrecogin alfa (activated) in the treatment of severe sepsis: the effect on hospital costs. Crit Care Med. 2002;29(suppl 12):Al. Abstract no. 4.

15. Sands KE, Bates DW, Lanken PN, et al. Epidemiology of sepsis syndrome in 8 academic medical centers. JAMA. 1997;278:234-240.

16. Ollendorf DA, Fendrick AM, Massey K, Williams GR, Oster G. Is sepsis accurately coded on hospital bills? Value Health. 2002;5:79-81.

17. ICD-9-CM Official Guidelines for Coding and Reporting.Centers for Disease Control. National Center for Health Statisitics. 2003. Available at: http:www.cdc.gov/nchs/data/icd9/icdguide.pdf. Accessed October 2003.

18. Motheral BR, Fairman KA. The use of claims databases for outcomes research: rationale, challenges, and strategies. Clin Ther. 1997;19:346-66. 